\title{
The in Vitro Osteogenic and Angiogenic Effects of Sodium- free Fluoride-containing Bioactive Glasses
}

DOI:

10.1016/j.matlet.2019.04.016

\section{Document Version}

Accepted author manuscript

Link to publication record in Manchester Research Explorer

\section{Citation for published version (APA):}

Lei, Z., Li, F., Chen, X., Mo, F-M., \& Chen, X. (2019). The in Vitro Osteogenic and Angiogenic Effects of Sodiumfree Fluoride-containing Bioactive Glasses. Materials Letters, 248, 138-142.

https://doi.org/10.1016/j.matlet.2019.04.016

\section{Published in:}

Materials Letters

\section{Citing this paper}

Please note that where the full-text provided on Manchester Research Explorer is the Author Accepted Manuscript or Proof version this may differ from the final Published version. If citing, it is advised that you check and use the publisher's definitive version.

\section{General rights}

Copyright and moral rights for the publications made accessible in the Research Explorer are retained by the authors and/or other copyright owners and it is a condition of accessing publications that users recognise and abide by the legal requirements associated with these rights.

\section{Takedown policy}

If you believe that this document breaches copyright please refer to the University of Manchester's Takedown Procedures [http://man.ac.uk/04Y6Bo] or contact uml.scholarlycommunications@manchester.ac.uk providing relevant details, so we can investigate your claim.

\section{OPEN ACCESS}




\section{Accepted Manuscript}

The in Vitro Osteogenic and Angiogenic Effects of Sodium-free Fluoride-containing Bioactive Glasses

Zhuo Lei, Fenghua Li, Xiaohui Chen, Fong-Ming Mo, Xiaojing Chen

PII:

$$
\text { S0167-577X(19)30564-6 }
$$

DOI: https://doi.org/10.1016/j.matlet.2019.04.016

Reference: MLBLUE 25998

To appear in:

Materials Letters

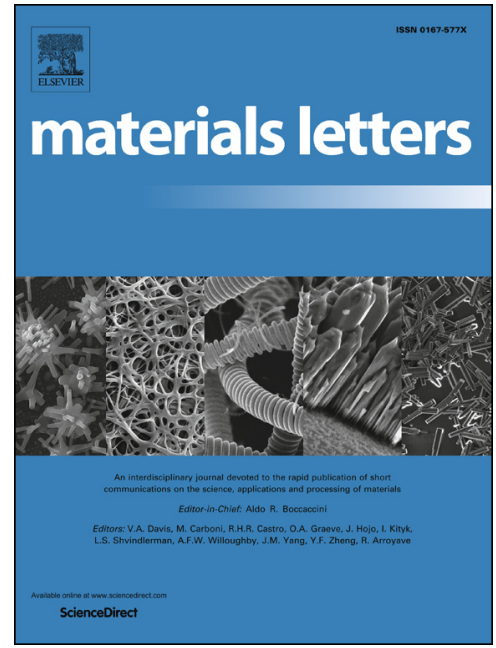

Received Date: 21 January 2019

Revised Date: $\quad 23$ March 2019

Accepted Date: $\quad 4$ April 2019

Please cite this article as: Z. Lei, F. Li, X. Chen, F-M. Mo, X. Chen, The in Vitro Osteogenic and Angiogenic Effects of Sodium-free Fluoride-containing Bioactive Glasses, Materials Letters (2019), doi: https://doi.org/10.1016/ j.matlet.2019.04.016

This is a PDF file of an unedited manuscript that has been accepted for publication. As a service to our customers we are providing this early version of the manuscript. The manuscript will undergo copyediting, typesetting, and review of the resulting proof before it is published in its final form. Please note that during the production process errors may be discovered which could affect the content, and all legal disclaimers that apply to the journal pertain. 
The in Vitro Osteogenic and Angiogenic Effects of Sodium-free Fluoride-containing Bioactive Glasses

Zhuo Lei ${ }^{\mathrm{a}}$, Fenghua $\mathrm{Li}^{\mathrm{a}}$, Xiaohui Chen ${ }^{\mathrm{b}}$, Fong-Ming $\mathrm{Mo}^{\mathrm{c}}$, Xiaojing Chen ${ }^{\mathrm{a}^{*}}$

${ }^{a}$ Xiangya Stomatological Hospital \& School of Stomatology, Central South University, Changsha, China

${ }^{b}$ Division of Dentistry, School of Medical Sciences, The University of Manchester, Manchester, UK

${ }^{\mathrm{C}}$ Key Laboratory of Nanobiological Technology of National Health and Family Planning Commission, Xiangya Hospital, Central South University, Changsha, China

*Corresponding author: xiaojing.chen@csu.edu.cn

\section{Abstract}

Fluoride is known to stimulate bone formation. Recently, novel sodium-free fluoride-containing bioactive glasses (FBGs) were found to be highly bioactive, dissolved in Tris and SBF buffer solutions releasing ions and promoting fluorapatite formation and offered great potential for medical and dental applications.

However, there is very limited knowledge on the biological effects of these glasses, which are extremely important for clinical applications. This study investigated the bioactivity of FBGs and their osteogenic and angiogenic effects on MC3T3-E1 osteoblasts in $\alpha$-MEM for the very first time. Results showed that these highly bioactive novel FBGs were non-cytotoxic. The addition of fluoride promoted mineralization, collagen formation, osteogenic and angiogenic genes expression in osteoblasts. Compared to sodium-containing BGs, a higher amount of fluoride can be incorporated into sodium-free BGs to improve glass biological effects.

Key words: Amorphous materials; Biomaterials; Sodium-free; Fluoride; Osteogenesis; Angiogenesis

\section{Introduction}

Bioactive glasses (BGs) are degradable materials, which can release ions and form an intimate bond with living tissues via the formation of an apatite-like layer [1]. Numerous in vitro [2] and in vivo [3] studies showed that BGs promoted bone regeneration. Some BGs induced high expression of genes related to osteogenesis and angiogenesis in osteoblasts and endothelial cells [4-6].

Customized glass properties tailored by changing glass compositions and structure are critical to fulfill different applications. Based on the well-accepted glass degradation mechanism proposed by Hench [7], the ion exchange between $\mathrm{Na}^{+}$from glass and $\mathrm{H}^{+}$from solution controls glass degradation and therefore glass 
bioactivity. However, a high sodium content makes BGs hydroscopic and less stable [8]. Recently, Chen and team [9] found no significant difference in degradation and apatite formation rates between BGs with and without the presence of sodium, suggesting that sodium might not be the key factor controlling bioactivity. Moreover, the absence of sodium avoided the uncontrolled $\mathrm{pH}$ increase resulting from ion exchange, which is normally unfavorable to cells.

Fluoride demonstrated great biological effects in stimulating bone formation [10] and fluoride-containing BGs exhibited anti-caries effect and osteointegration [11]. Adding appropriate amount of fluoride into sodium-free BGs reduced the increase of $\mathrm{pH}$ for the immersion solutions and promoted rapid glass degradation and the formation of more acid-resistant fluorapatite in vitro [12,13] making those FBGs attractive for medical and dental applications. However, there was no study on the biological effects of FBGs. This paper aimed to discover the osteogenic and angiogenic effects of FBGs to provide essential information for a better understanding and exploitation of these glasses for clinical applications.

\section{Material and Methods}

\subsection{Glass synthesis and BG-conditioned media preparation}

A FBG series $\left(\mathrm{SiO}_{2}-\mathrm{P}_{2} \mathrm{O}_{5}-\mathrm{CaO}-\mathrm{CaF}_{2}\right)(\mathrm{Table} 1)$ were synthesized by a melt-quench route as described previously [14]. The glass bioactivity in $\alpha$-MEM was evaluated according to the method described by Chen et al. [9]. After different immersion times (1,3,6 and 9h), BG-containing $\alpha$-MEM was filtered into solution and solid. The concentration of calcium, phosphorus and fluoride in the solution was measured using ICPOES and fluoride ISE. The solid collected after $9 \mathrm{~h}$ immersion was analysed using FTIR. The filtered $3 \mathrm{~h}$ BGcontaining $\alpha$-MEM solution was chosen for the biological effects in this study. $100 \mathrm{U} / \mathrm{ml}$ penicillin and 100 $\mathrm{mg} / \mathrm{ml}$ streptomycin (pen-strep) were added to produce BG-conditioned media [11].

Table 1. Glass compositions in mol\% [14]

\begin{tabular}{lllll} 
Glass code & $\mathrm{SiO}_{2}$ & $\mathrm{CaO}$ & $\mathrm{P}_{2} \mathrm{O}_{5}$ & $\mathrm{CaF}_{2}$ \\
\hline GPF0.0 & 38.1 & 55.5 & 6.3 & 0.0 \\
GPF3.0 & 37.0 & 53.9 & 6.1 & 3.0 \\
GPF4.5 & 36.4 & 53.0 & 6.0 & 4.5
\end{tabular}




$\begin{array}{lllll}\text { GPF6.0 } & 35.9 & 52.2 & 5.9 & 6.0 \\ \text { GPF9.3 } & 34.6 & 50.4 & 5.7 & 9.3\end{array}$

\subsection{Cytotoxicity and Alkaline phosphatase (ALP) activity}

MC3T3-E1, the mouse osteoblast-like cell line (Chinese Academy of Sciences) was cultured in $\alpha$-MEM supplemented with $10 \%$ FBS and $1 \%$ pen-strep. The cytotoxicity of BG-conditioned media was evaluated by MTT assay. ALP activity was assessed by enzyme histochemical assay [11].

\subsection{Mineralization, collagen formation and Gene Expression}

The mineralization and collagen formation abilities of FBGs were quantified by Alizarin Red S staining and Sirius Red Staining. Cells were cultured for 14-21d in an osteogenic medium (BG-conditioned medium with $50 \mathrm{ug} / \mathrm{ml} \mathrm{L-ascorbic} \mathrm{acid} \mathrm{and} 5 \mathrm{mM} \beta$-glycerophosphate). The plates were stained with $2 \%$ Alizarin Red S or Sirius red solution accordingly [11]. The OD values were measured at 560nm.

The osteogenic and angiogenic gene expression of cells treated for 7 and 14d was investigated by qRT-PCR as described previously [11]. All primer sequences were listed in Table S1.

One-way ANOVA was used to perform statistical analysis. Results were shown as mean \pm standard deviation.

\section{Results and Discussion}

\subsection{Ion release and apatite formation}

The measured concentrations of calcium, phosphorus and fluoride are result of ions presented in control culture media, ion released from glass and that consumed for apatite formation. All studied BG conditionedculture media showed similar trend in the measured calcium content which clearly increased in the first $3 \mathrm{~h}$ followed by minor changes (Fig.1a). The phosphorus concentration exhibited a general reduction for all glasses (Fig.1b), which was a consequence of the consumption of phosphorus for apatite formation [14]. Compared to other glasses, GPF9.3-conditioned medium showed a lower phosphorus concentration, which is probably attributed to the partially crystalline nature of GPF9.3. The crystallized apatite suppressed glass degradation while promoted further apatite formation via a seeding effect [13]. A clear increase of fluoride 
concentration was found in the first $3 \mathrm{~h}$ while a decrease was evident between 3 and $6 \mathrm{~h}$ for all compositions (Fig.1c). The ion release profiles presented (Fig.1a-c) indicated that all the studied glasses are highly bioactive and apatite formation became the predominant process after $3 \mathrm{~h}$ immersion.

FTIR spectra (Fig.1d) of glasses after 9h immersion in $\alpha$-MEM also proved rapid glass degradation and apatite formation. Compared to the spectra of the non-immersed glass powders shown in a previous study [13], the intensity of non-bridging oxygen $\mathrm{Si}_{-} \mathrm{O}^{-}$band at $920 \mathrm{~cm}^{-1}$ decreased significantly after immersion, while the crystalline orthophosphate band around $1035 \mathrm{~cm}^{-1}$ clearly sharpened. The typical apatite split bands at $600 \mathrm{~cm}^{-1}$ and $560 \mathrm{~cm}^{-1}$ were visible for FBGs with higher fluoride content.

a

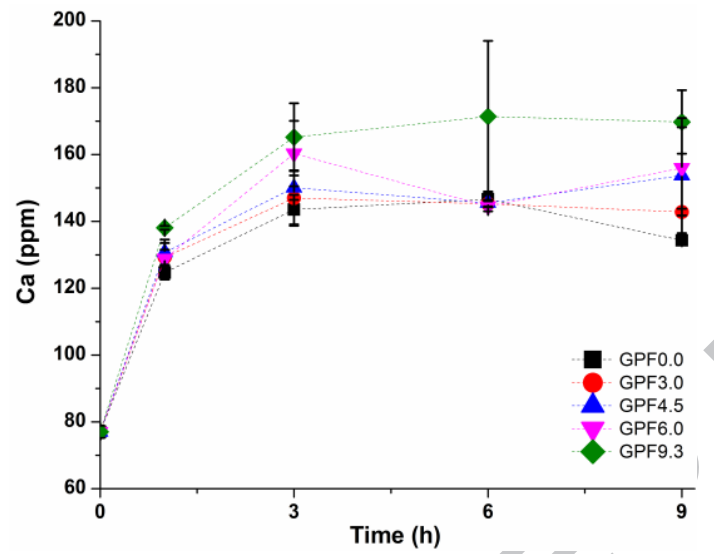

C

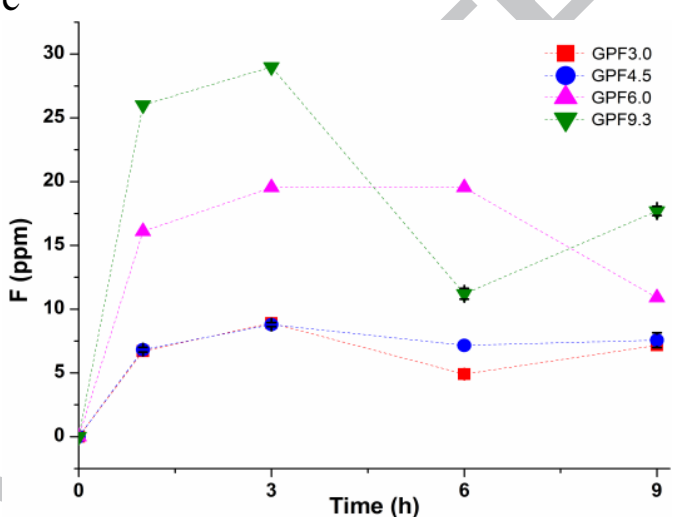

b

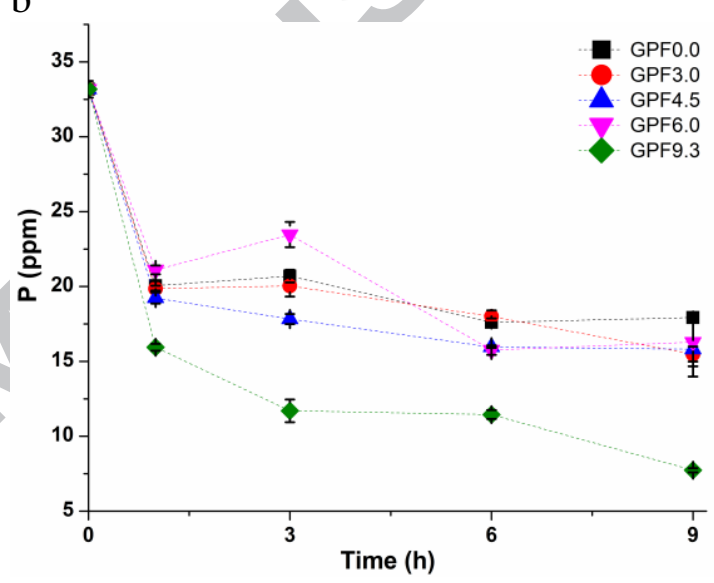

d

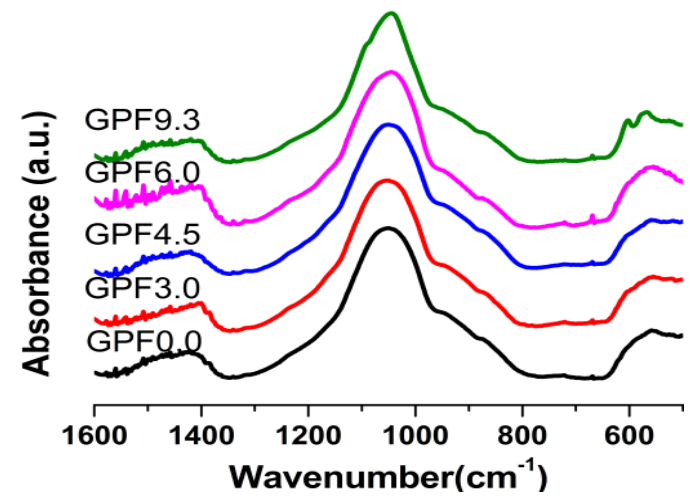

Fig. 1. Measured ion concentration in FBGs conditioned $\alpha-M E M$ a) calcium, b) phosphorous and c) fluoride at 1h, 3h, 6h and 9h; d) FTIR spectra of glasses after $9 \mathrm{~h}$ immersion in $\alpha-M E M$.

\subsection{Cell viability and ALP activity}

After 4 and 10d in culture, cells treated with culture media containing ions released from the BGs after $3 \mathrm{~h}$ immersion showed a greater cell viability than that treated with control culture medium. Additionally, the viability was significantly higher in MC3T3-E1 treated with GPF3.0 and GPF4.5 extracted culture media. 
Similar effect has been previously shown in sodium- and fluoride-containing BGs (NaFBGs) studied by Liu et al. [11]. After 7d in culture, apart from cells treated with extracted GPF6.0 and GPF9.3 media that exhibited clearly lower viability, the viability in cells treated with FBGs showed no significant difference from control, indicating that the extracted products of FBGs were mostly non-cytotoxic.

After 4d in culture, the ALP activities of MC3T3-E1 cultured with BGs media showed no significant difference to control (Fig.2). However, after 7 and 10d in culture, the extracted BGs-culture media were found to reduce the ALP activity. The more the fluoride presented in glass, the lower the ALP activity. This is in agreement with the NaFBGs studied in [11], which showed that the ALP activity was suppressed with fluoride content increased from 1 to $5 \mathrm{~mol} \%$.

\subsection{Mineralization and collagen formation}

The results of the mineralization ability of FBGs quantified by Alizarin Red S staining (Fig.2c) showed that the MC3T3-E1 cells cultured with extracted BGs media exhibited significant increase $(p<0.05)$ in the staining compared with that in control medium after both 14 and 21d. Glasses GPF0.0, GPF4.5 and GPF6.0 demonstrated the highest mineralization ability by $21 \mathrm{~d}$, suggesting that fluoride may interact with mineralized tissues in a dose dependent manner. Similar effects were also shown in NaFBGs [11]. The quantitative and qualitative results of Sirius Red staining showed that BG-conditioned media dramatically stimulated collagen formation after both 14 and $21 \mathrm{~d}$ in culture compared to the control groups (Fig.2d). This effect was more obvious $(\mathrm{p}<0.05)$ in the FBGs $\left(\mathrm{CaF}_{2}<9.3 \mathrm{~mol} \%\right)$, while GPF3.0 and GPF4.5 showed the most significant effect, indicating that a moderate amount of fluoride $(<9.3 \mathrm{~mol} \%)$ in the sodium-free BGs modulated the collagen formation ability of MC3T3-E1 osteoblasts. Similar regulating effect was found in sodium-containing BGs [11] with a small amount of fluoride ( $\leq 3 \mathrm{~mol} \%)$, while a suppression effect of collagen formation was seen in the glass with $5 \mathrm{~mol} \%$ fluoride. Therefore, a conclusion can be made that the favorable amount of fluoride to promote mineralization and collagen formation is different in NaFBGs and FBGs. Less fluoride was favored in NaFBGs compared to FBGs.. However, the incorporation of higher fluoride content improved glass properties, such as reducing glass melting temperature, glass transition temperature and the increase in $\mathrm{pH}$ after glass degradation [15]. Therefore, in the context of having high 
bioactivity, the glass without the presence of sodium may be more beneficial to maximize the benefits of adding fluoride.

a

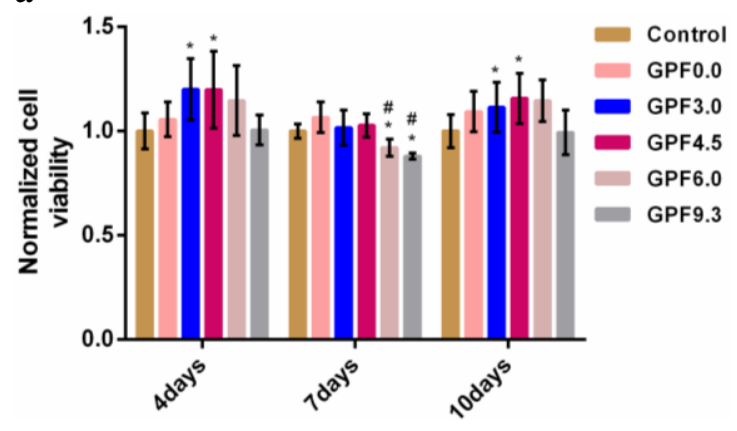

c
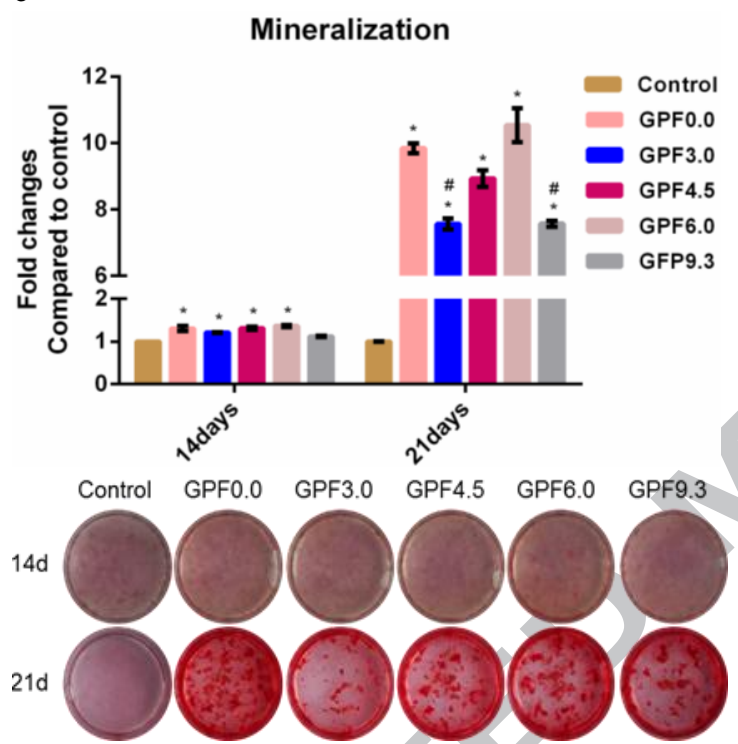

b

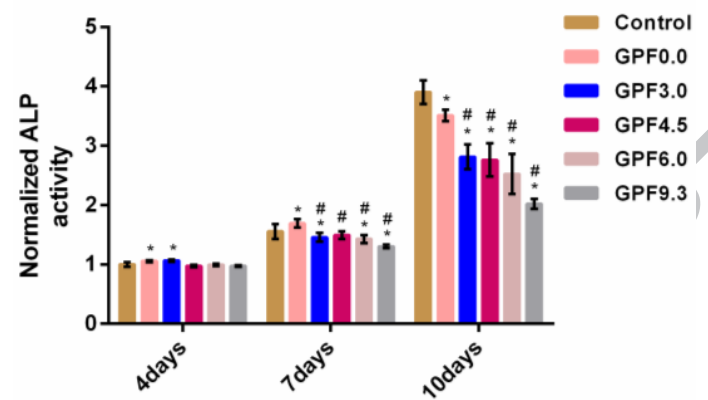

d
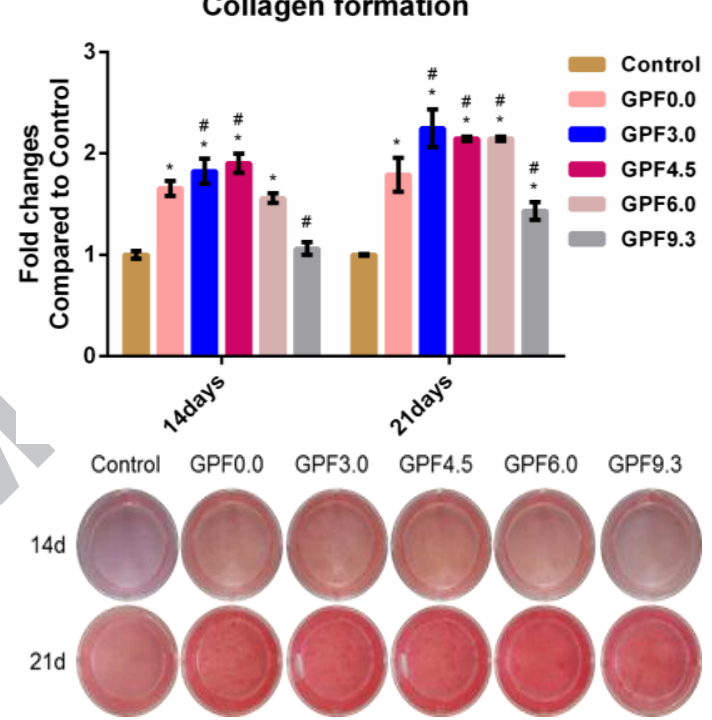

Fig. 2. a)Cell viability, b)ALP activity of BG-conditioned media on MC3T3-E1; Quantitative and Qualitative results of MC3T3-E1 c) mineralization in BGconditioned media and d) collagen formation in BG-conditioned media. Results showed as a fold change compared to the control group. ${ }^{*} \mathrm{P}<0.05$, compare with control group; \#P<0.05, compare with GPF0.0.

\subsection{Gene expression}

After 7d culture, the expression of the osteogenic genes BMP-2 and OPN investigated by qRT-PCR showed either higher or no significant difference for BGs treated groups compared to the control group (Fig.3a, b), while a lower expression was seen for Col1a1. However, after 14d culture, a significant up-regulated effect on all BMP-2, OPN and Col1a1 gene expression was found in GPF4.5 and GPF6.0 relative to the control suggesting that a moderate amount of fluoride contributed to bone formation at $14 \mathrm{~d}$ (Fig.3c). This is in accordance with the finding based on ALP and staining results (Fig.2).

VEGF stimulates the formation of blood vessels and it is essential for bone formation [16]. Compared to 
control, the expression of VEGF was higher in BGs groups, especially for FBGs at 7d (Fig.3d). Silicon was found to stimulate VEGF secretion and promote angiogenesis [17]. In this study, similar amount of silicon ions was detected in $\alpha$-MEM after glass immersion (Fig.S1), which regulated the expression and secretion of VEGF secretion therefore facilitating angiogenesis [17]. It was shown that glasses GPF4.5 and GPF6.0 have remarkable effects, while GPF9.3 was less effective, indicating that fluoride upregulated VEGF gene expression synergetically with silicon in a dose-dependent matter. Moreover, the phosphorus content in media might also modulate osteogenic and angiogenic genes expression, since GPF9.3 was partially crystallized and less phosphorus was present in GPF9.3 conditioned-culture media. . There is no obvious difference in VEGF expression between 7 and 14d for glasses, except GPF4.5 and GPF6.0. However, in the NaFBGs [11] VEGF gene expression enhanced from $7 \mathrm{~d}$ to $21 \mathrm{~d}$ in culture for all the compositions. This difference might be due to the fact that FBGs degraded very rapidly [14], angiogenesis may occur earlier than that in NaFBGs. Additionally, sodium might also be involved in VEGF regulation, which needs further investigation.
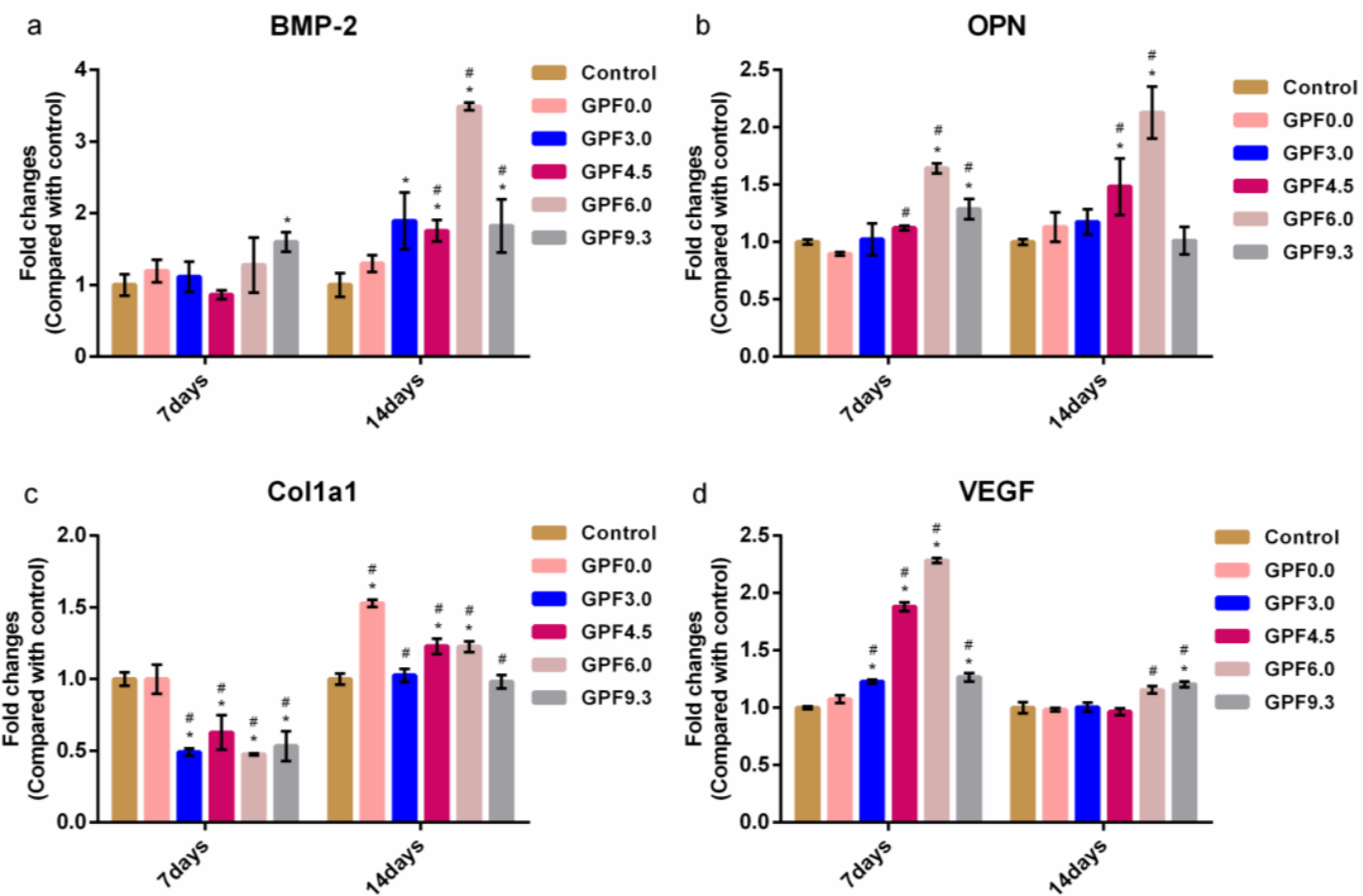

Fig. 3. The expression of (a)BMP-2 gene, (b)VEGF gene, (c)OPN gene, (d)Col1a1 gene detected by qRT-PCR and presented as a fold change compared with the control. * $\mathrm{P}<0.05$, compare with control group; $\# \mathrm{P}<0.05$, compare with GPF0.0. 


\section{Conclusions}

The studied FBGs are highly bioactive in $\alpha$-MEM culture media and non-cytotoxic. Compared to NaFBGs, a higher amount of fluoride can be incorporated into FBGs for improving biological effects. The incorporation of a moderate amount of fluoride (about 4.5 and $6.0 \mathrm{~mol} \% \mathrm{CaF}_{2}$ ) into sodium-free $\mathrm{BGs}$ enhanced the mineralization and collagen formation abilities of MC3T3-E1 osteoblasts, meantime activated their osteogenic and angiogenic genes expression. Thus, these FBGs are attractive for bone substitutes.

\section{Acknowledgements}

This study was supported by National Natural Science Foundation of China (81701015) and the Fundamental Research Funds for the Central Universities of Central South University (1053320170033).

\section{References}

[1] L. L. Hench, J Mater Sci Mater Med 17 (2006) 967-978.

[2] E. Verne, et al.,Biomaterials 26 (2005) 5111-5119.

[3] Y. Fujishiro, L. L. Hench, H. Oonishi, J Mater Sci Mater Med 8 (1997) 649-652.

[4] Kargozar S, et al.,Trends in Biotechnology 36 (2018) 430-444

[5] H. Li, et al., Biomaterials 35 (2014) 3803-3818.

[6] J. Will, L. C. Gerhardt, A. R. Boccaccini, Adv Biochem Eng Biotechnol 126 (2012) 195-226.

[7] L. L. Hench, J. Wilson, An introduction to bioceramics, 1993, p. 386.

[8] Z. EC, Quim Nova 21 (1998) 356-360.

[9] X. Chen, et al., Int J Appl Glass Sci 8 (2017) 428-437.

[10] L. Huo, et al., Biol Trace Elem Res 155 (2013) 142-149.

[11] J. Liu, et al., Dent Mater 32 (2016) e221-e237.

[12] M. Mneimne, et al., Acta Biomaterialia 7 (2011) 1827-1834.

[13] X. Chen, et al., Journal of Non-Crystalline Solids 402 (2014) 172-177.

[14] X. Chen, et al., Materials 7 (2014) 5470-5487.

[15] D. S. Brauer,et al., Acta Biomater 6 (2010) 3275-3282.

[16] J. M. Kanczler, R. O. Oreffo, Eur Cell Mater 15 (2008) 100-114.

[17] A. A. Gorustovich, J. A. Roether, A. R. Boccaccini, Tissue Eng Part B Rev 16 (2010) 199-207. 


\section{Highlights:}

- The studied Na-free F-containing glasses are highly bioactive and non-cytotoxic.

- The biological effects of Na-free F-containing glasses were studied first time.

- F promoted mineralization, collagen formation and genes expression in osteoblasts.

- More F can be added into Na-free bioactive glasses to enhance biological effects. 